MATEC Web of Conferences 52, 03002 (2016)

DOI: $10.1051 /$ matecconf/20165203002

(C) Owned by the authors, published by EDP Sciences, 2016

\title{
In-Vitro Design Protocol: Artificial Situation Strategy Uses to Comprehend Designers' Thought
}

\author{
Rusmadiah Anwar ${ }^{1,3, a}$, ShahrimanZainal Abidin ${ }^{1,2}$ and Oskar Hasdinor Hassan ${ }^{3}$ \\ ${ }^{1}$ Formgiving Design Research Group, Faculty of Art \& Design, UiTM, 40450 Shah Alam, Malaysia \\ ${ }^{2}$ Research Innovation Business Unit, UniversitiTeknologi MARA, 40450 Shah Alam, Malaysia \\ ${ }^{3}$ Department of Industrial Ceramic, Faculty of Art \& Design, UiTM, 40450 Shah Alam, Malaysia
}

\begin{abstract}
The challenge of current trends in design research and point out some of their activities, such as the gap between aesthetic and technical need, and the chasm between ambiguous and quantified in design practice. So, it is hard to surprise the product designers fail to combine a systematical methodology in analyzing design activity. This paper will assess the comprehensible and abilities of In-Vitro Design Protocol (IVDP) in mapping and analyzing design activities. This paper thus attempts to provide a systematic design investigation to researcher and discuss a few key demands on such foundation, include the capture and monitoring used for observational studies in artificial environment strategy.
\end{abstract}

\section{Introduction}

Recording design activity among creative designer through video becoming more popular, and define as primary field materials that treat as collecting inquiry data [1] [2]. Even this empirical research has widely practiced among psychologist include engineering designer, it is, however, difficult to find related research involve a kind of research in art and design practices. The interesting about this evidence-based data is, it allowed us to investigate the design thought among chosen subjects empirically. It also brings a mystery outcome during the experiment, which unexpected before. In Valkenburg's recorded videotape and written transcript has shocked by the amount of incomprehension design content among the subjects [3]. If a kind of systematic design methodology such engineering can bring unexpected results, we believed, more intuition design thought from creative designer could be captured and recorded through empirical research. Giving great design behavior and the knowledge gap to be described, particularly in the early phase (the design activity) of any industrial design practices. Where in this stages, the form of a product is poorly supported, mostly at the stage of conceptual [4]. To select a valuable conceptual design, the solution searching process should begin with a divergent until convergence step [5-7].

Conventional models by Cross describe a traditional design process attempt to build prescriptive models of the design process [8]. Cross mostly disagrees with the generation of solution concept identified by these existing models and discovered plenty of examples of the excellent solution provided in a wrong direction. Therefore, Cross suggests an essential structure to the design process of analysis-synthesis-evaluation that not followed the conventional design practice. It was emphasizing

\footnotetext{
${ }^{\text {a }}$ Corresponding author : rusma935@salam.uitm.edu.my
} 
on the performance specifications logical derived from the design problem, generating several alternative design concepts by building-up the best sub-solution and making a rational choice of the best alternative design. This process has same interpretation with "goal-oriented problem-solving activity" [9]. It defines as 'the goal' plays a huge influence any goal-oriented activities. Therefore, the success of any design development depends on the particular establishment at the beginning of the 'real' project goals. Consequently, completeness of design depends on the ability to introduce the legitimate objectives. In a similar point of view, Pugh define that industry was aware of 'total design'; "the systematic activity necessary for the identification of the market/user need, to the selling of the successful product to satisfy that need an activity that encompasses product, process, people and organization" [10].

\section{A review of design research related to design activity}

Design research is systematic inquiry whose goal is knowledge of, or in, the embodiment of configuration, composition, structure, purpose, value, and meaning in man-made things and systems [11]. However, in current art and design practice, there has been increasing debate about research as a discipline in art and design [12]. Neither artist nor designer is regularly undertaking formal research based on existing research method from any Physical and Social Sciences. Conversely, it does not convey to any of process correctly. Do the art activity (painting, sculpture, printmaking, etc.) involves 'design' or, any design development include 'art' (color, line proportion, scale, etc.) in the practice? It is the nature of art and design and becoming one of the topics for such debates. However, industrial design is not art, craft or engineering; it has it own purpose, values, measures and procedure [13]. Looking at design research from the design methodology and design science perspectives in a sense for such topic, it is principally (five elements of design research) tries to answer the obligation of design to the humanities [14]. It shows that design research only focuses in comprehending the methodology, thus, metaphor structure is neglected and overlooked. The reasons Bayazit describe the elements in design research as his disagreement with the art, craft and design people call what they do for art and design "research." Practicing activities when creating a work of art or craft cannot be considered research [14]. However, somehow rather, he agrees that the observable phenomena are the research gap for the external observer to do research into how an artist is working with their creative works.

In our point of view, most of the design activity traditionally is sketch neither line nor form, the construction can totally consider as "research," especially when the activity involves design process. Design-inspire approaches are crucial in the form development process [15]. Designers generate metaphors by captivating attributes from one or more entity before transforming it to product [16]. These inspirations could come from new ideas from designer's previous knowledge and experiences [17]. Designers use metaphor as a strategic approach to design activity [18] in which, metaphors is one of the mechanisms that permits the well-known mental imagery of subjective experiences and judgments based on general experiences that could be neutrally linked [19]. The metaphorical element is important, as it frequently serves as ways in stimulating designer's creativity in the design process. As an artifact that reveals something insightful, conceptual metaphors are used in relating the connotation of separated entities to be conveyed and comprehended, thus allowing creativity during form development of a product [16]. The metaphors also contribute in assisting in different thinking by bridging different domains in design problem solving [20-21].

To make possible for an external observer able to do research into how the artist or "creative" [2223] designer's thinks, we use an image to create the link (especially on the meaning) between ideas. The interpretations of design research as defined [14], inclusive with a visual metaphor we have suggested are summarized in Figure 1. Tea brewing machine choose as subject to link with Cross's three main categories that involve design research with people, process, and product [24]. The coherent examples of tea brewing machine have employed successively in examining the two structure variation methods [25]. This variation of relative arrangement defines as 'quantified structure' offer a final choice of criteria looked-for by considering the most promising structure [26]. 
Based on Figure 1, we can realize the topic of design research mostly related to design activity as defined by Cross. Metaphorically, the design praxeology can be studied into the topic of A, B and E; Design phenomenology study suit with the issue of C and D. Even though topic B is the only design research can fall into epistemology design categories, the same argument (B) has fallen into design praxiology categories. It confirms that discussion in the design process (design praxiology) by the designer (design epistemology) required in-depth investigation. Subconsciously answered the practicing activities when performing creative work can be considered as research. The structure of empirical study based on creative designers will explain in the next chapter.

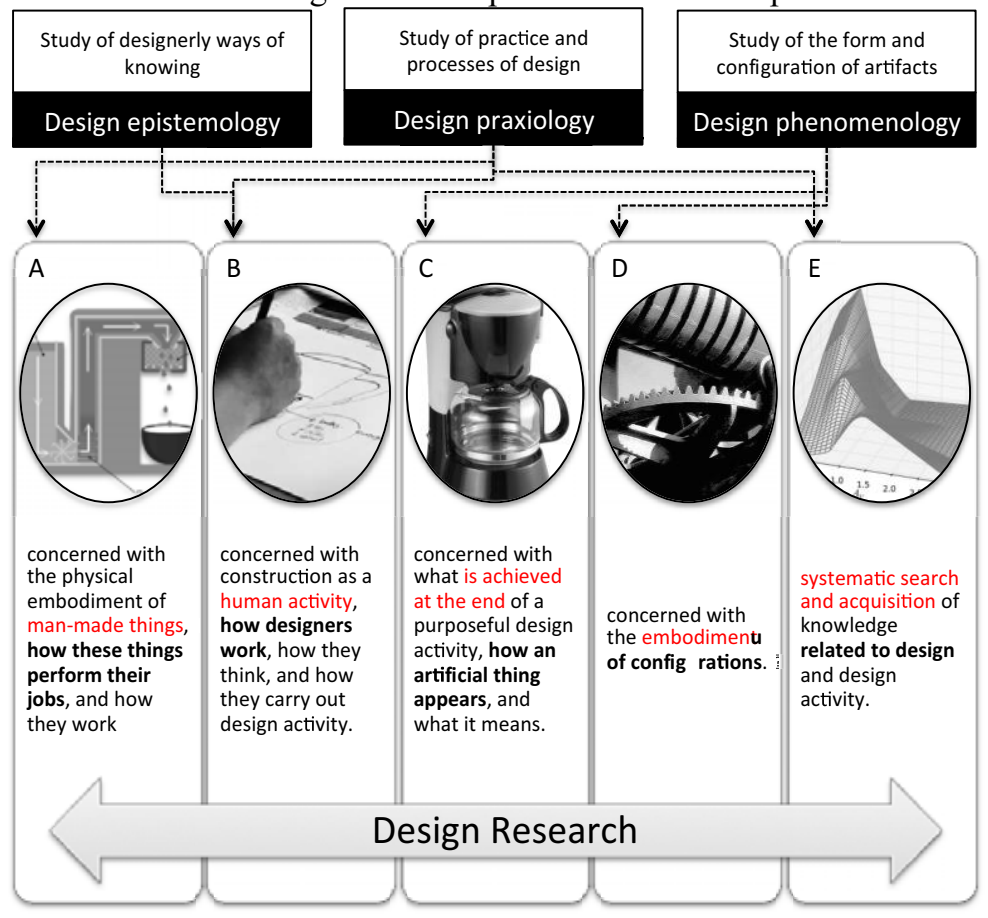

Figure 1. Designer's strategic metaphor design language linked to design research.

\section{Organizing in-vitro design protocol as investigation format strategy}

As per discussed in Section 2, design grows as a discipline with its research base and Cross believe, there will be a growth in the number of emerging designer-researchers. To devise an appropriate standard or addition to a methodology, it is necessary to identify the 'designerly' ways of knowing, thinking and acting [24]. However, there is no coherent theory exist in current literature about characteristics of metaphorical form in product design embedded based on the representation of mind by designers' from the perspective of Islamic influences. Thus, it become an advantages of this study: (i) to uncover how designers interpret metaphorical form based on Islamic influences, (ii) to understand the influence of incremental, radical design that changes respective to preceding designs, and (iii) to confirm the similarities of characteristics of metaphorical form representation in the context of syntactic that can be generalized as a brand and identity for Islamic product design. We suggest in-vitro design protocol (IVDP) to investigate the existence of metaphorical understanding instead of structure principal during design development. A video observation based on protocol design analysis of designer sketching activities by practitioners conducted and strengthened by a semistructured interview with an expert on the evaluation of selected product architecture in the analysis of representation concerning metaphorical form syntactic in design. The following steps will describe the fragmentation of activity as the primary structure for this IVDP. 
STEP 1: Understanding of design taxonomy (the task).Designers may sketch only to a partial degree ("completeness") in metaphorical form process. How, then, are elements metaphorical form by designers on completeness? [27]. This question close to the design models summarize by Blessing through the typology of design models as stage-based and activity-based [28], which realized the models that concerned with morphological and problem-solving dimension. Furthermore, the model prescribing well-structure activities within stagesand signify to convergence on a design solution where concrete activities within a stage used progressively [28]. The models presented will be as a benchmark for the comprehensively understand the design thought during the experiment.

STEP 2: Time framing (the design abstraction limitation). Even though the typology of design model able to describe the potential convergence to a design solution, designer (after this call 'subject') involves in this IVDP been control by time duration. The task given is to identify as much as solution ideas as possible. Including the selecting multiple choices of ideas that should be considered during design activity [29]. As design activity classified and not think as research due to the demanding reasonable research outcomes while performing an artwork or design work, we decide to bring a "short-term memory" (STM) in this design protocol. The reason to carry STM in IVDP is to investigate how possible the data inquiry during design activity is constructed in a short period. It will be a part of completing goal-targeted action among designers. Tjalve rationalizes the search for examination of solution, evaluation and choice of shows in the theory of search for and examination of solution, evaluation and choice as the characteristic sequence in product synthesis [26].

STEP 3: Conducting design protocol analysis (the experiment). The final formats for IVDP as investigation strategy following the design protocol methodology proposed by Anwar [30]. The issues on matters of the complexity of design research studies, including the difficulty of disentangled the relationship connection, it is important to adopt the same protocol to bring a wider pattern of observation strategy (see Figure 2a). It is an important method to empirically creative designers thought; four cameras (views), influence panel (optional four), clock (time recording), work-space (designer's working area) and sketch book (design activity). The divergent convergentof design brief strategies as visualised in Figure 2ballow us retaining the experiment design format.

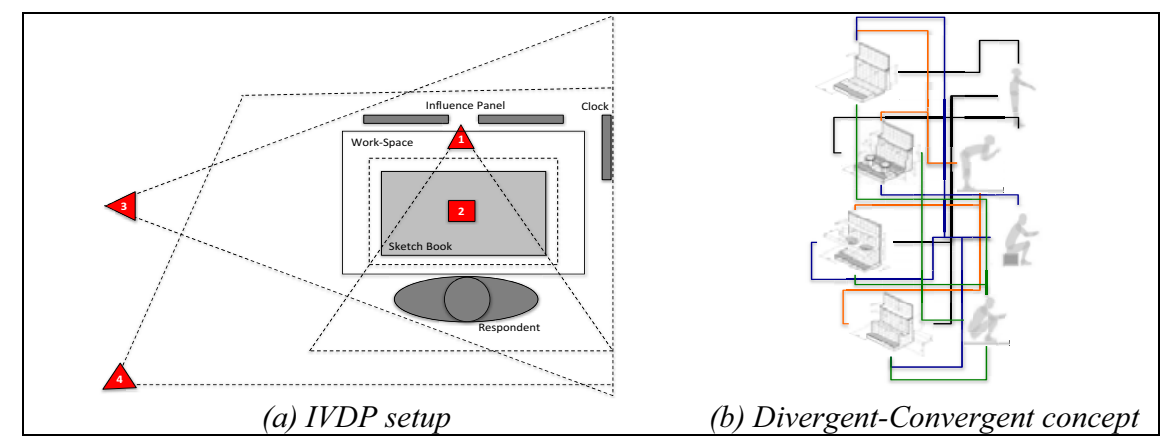

Figure 2.Controlled experimental and design protocolreplicated from Anwar et al. [29].

\section{The procedure of function means analysis as design strategy}

The Ablution Function Mean Analysis [7] set as a guideline on how designers practically apply while constructing form design. The design brief emphasized to analyze and evaluate the formgiving design in a variety of solution. This format is used to be as a document to integrate the design task of constructing Muslim's ablution product concept. Here, subject (designer) must allow to select all four ablution principles, perform on two combinations of four models mentioned with optional of two human posture (see Fig. 2b). These selections decide based on the primary ablution requirement [31]. In this guideline, the selection process known as Concept Integration Approach. This strategy aims to visualize explicitly design approaches through morphology analysis process concerning manual sketching. 
We also consider the limitation of time for the subject to develop a concept in short time procedure. As Abidin discover the designers typically use to choose what element to morph rather (identify as "selectivity") than transforming consistently [32], we set four panels (see Table 1) as an influence on the subject to 'spark' their ideas. It has divided into four groups; (Panel 1) Icon, Sign, and Symbol; (Panel 2) Object and artifact; (Panel 3) Building and Architecture; and (Panel 4) Culture and Heritage. The selected image for all panels is based on "public domain" with all images signify to Islamic characteristic. The purpose of this influence panel is to identify rather this picture will sketch in explicit or implicit ways. To investigate what expression portrays, the subject is asked to reflect on the question we provide. The following section determines how designer think in such a way introducing a basic structure for a product.

Table 1.The influence panel

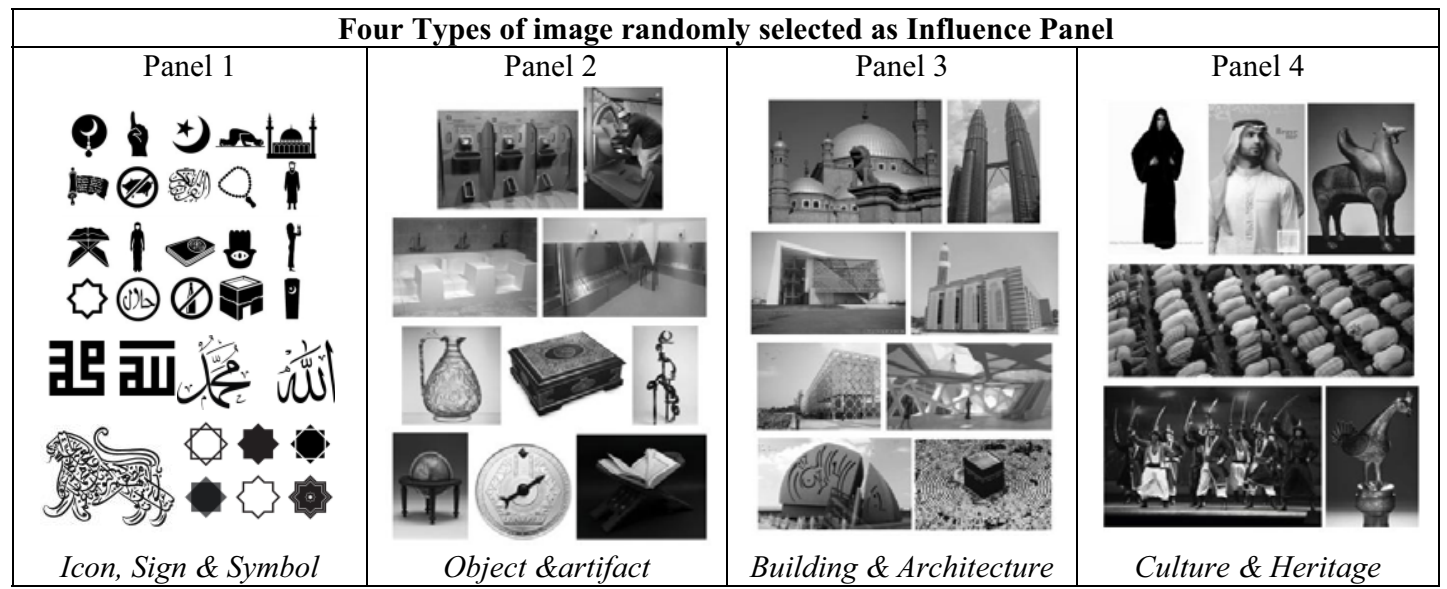

\section{Analysing designer's thought throughout IVDP experiment}

Experiencing the IVDP as illustrated in Figure 3, the ambiguous characteristics of metaphorical form through designers sketching processes of Islamic product design has lead to a natural variety in output. Whereas Abidin refer this phenomenon as "consistency." Thus, how do designers assess metaphorical form through their sketching assignments has discovered [18]. Throughout thirty minutes of design activity (short-term memory), this empirical study stipulates in-depth qualitative data reflected all artificial situations that have been arranged.

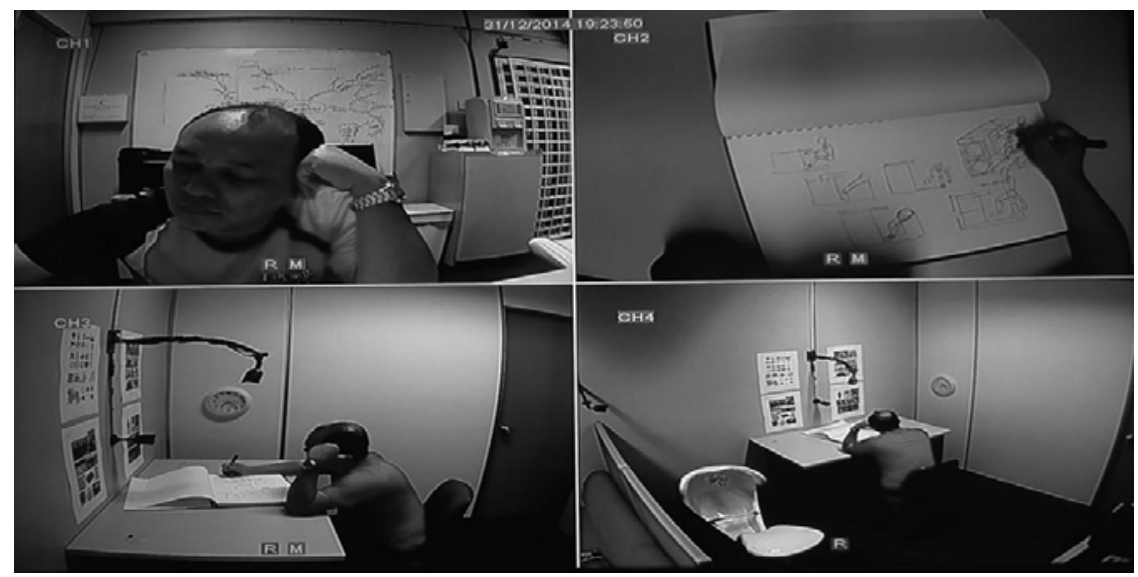

Figure 3.Expert designer as 'subject' during IVDP experiment. 
At the beginning of design activity, the subject often uses morphological analysis as similar with Funtion-Means Analysis Chart (See Table 2). A morphological chart was originally developed as a design method, overviews of function considered and representation produce especially on the product design perspectives. The morphological analysis has allowed subject to analyze the development procedure of ablution design concept. From here, the subject used to "trace out" potential whole system concept solution. Table II shows how the subject accomplishes the analysis by referring the Function Means Analysis for Ablution.

Table 2. The Function Means Analysis chart applied by the designer during the creation of ablution design [7]

\begin{tabular}{|c|c|c|c|c|}
\hline \multicolumn{5}{|c|}{ Function - Means Analysis Chart } \\
\hline Function & & & Means & \\
\hline Ablution Principle & Face & Both hand & Forehead & Both Feet \\
\hline Body Posture While Ablution & Stand & Bend & Sit & Squat \\
\hline Faucet design & Lever-type & Single-hole & Center-set & Wall-mount \\
\hline Water fall method & Flush (Pipe) & Dipping (Pond) & Scoop (Bailer) & Bailer \& Dip (Pond) \\
\hline Drain System & Open & Covered & Pipe & Trap \& pipe \\
\hline
\end{tabular}

IVDP experiment allows us to identify that designer able to derived design and divide three different levels of abstraction, which is abstract, semi-concrete and concrete. On the first level of design development (Table 3a), the subject seems to illustrate the form construction by tracing out non-priority means for ablution. Remaining means offer an initial and reasonable idea in realizing the concept. Structure design brief by stating the direction of design task in an apparent divergent phase, acknowledge by subject to present an excellent level of convergent. From the early (abstract level) process of design concept, the subject has determined the visou-spatial characteristics [33] of the ablution product. The form structure and configuration completely close to "higher order form elements' [34]. As refers to the abstract level, the subject has "assemble" all form element into one structure, with the inclusive of Islamic (arabesque) characteristic. Undergoes to the next level (semiconcrete) of a design process, the subject chose to implement a quantified structure strategy (See Table $3 \mathrm{~b}$ ) to determine the best consideration and most promising design structure for ablution practice.It comes to the end of design activity (concrete level) as subject confidently configures the form element with the relation of ablution requirements. In Table $3 \mathrm{c}$, though the product development, the subject has determined a gestalt study [35]. The ablution design structure organized, arranged and correlated well with the requirement of ergonomic, technical and practical of ablution performance requirements.

Table 3. The abstraction level of design experiment during IVDP

\begin{tabular}{|c|c|c|}
\hline \multicolumn{3}{|c|}{ The Abstraction Levels } \\
\hline (a) Abstract & (b) Semi-concrete & (c) Concrete \\
\hline $\begin{array}{c}\text { The assembly of form } \\
\text { elements. }\end{array}$ & Quantified structure of form elements. & $\begin{array}{c}\text { The configuration of form } \\
\text { elements. }\end{array}$ \\
\hline
\end{tabular}




\section{Discussions}

In this research, the design activity performed by creative designer able to give a contribution to the body of knowledge, especially in exploring how creating a craftwork (design) canrelateto the field of design research. It has proven in anyabstraction (abstract/semi-concrete/concrete)level of the design process has metaphorically give a significant meaning as research investigations. As the IVDP experiment is artificially set as design situation to provoke designer in constructing a form, the subject spectacularly drives the way of design think and approach into 'Formgiving Design Principle Solution' as a way for problem-solving. Transforming the whole design process into a concrete idea development stages. As 'Product Architecture' is a method whereby; the functional elements of a product will be assigned to its physical building blocks. Moreover, it can be implemented as early as idea development takes place, through sketches, function diagrams, and early sketch models [36]. So we refer to the experiment (Divergent Convergent Process) through IVDP as the first stage of next investigation strategy (product architecture) take place. IVDP has offering design investigation format, and it recalls on how practicing activities by a creative designer can considered as systematic research and given a cues to support another research phase.

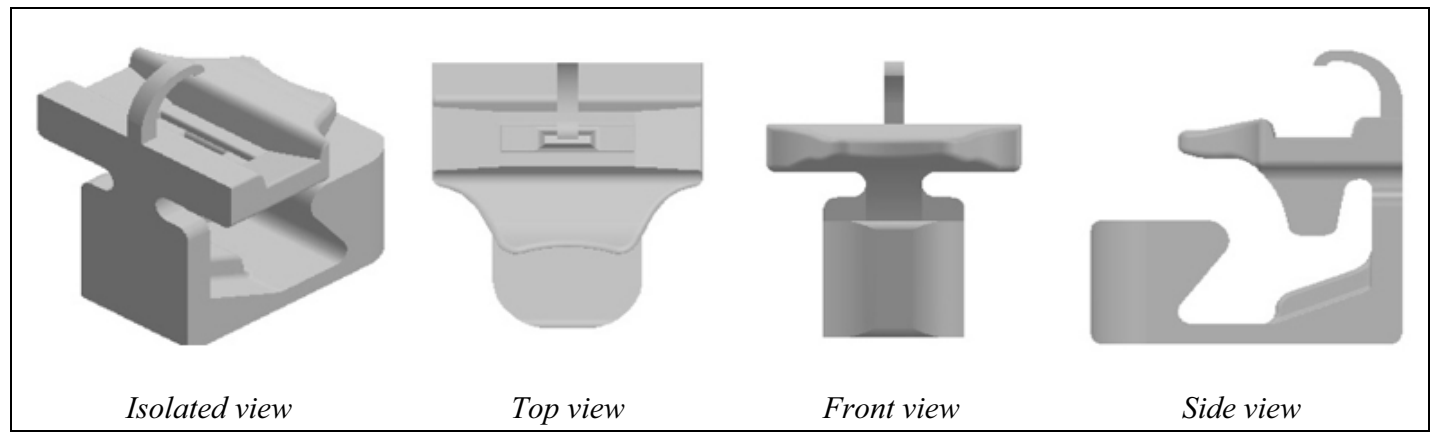

Figure4.The conceptual and embodiment form element of ablution throughIVDP experiment.

The experiment mean to focus on Divergent Convergent Process strategically to develop idea inspiring complete form of ablution concept (Figure 4). Throughout subject's 'function means analysis', we construct completely prefabricated idea (form) to make possible the concrete idea transformation through abstraction level (see Table 3) as a sample of a structure to attempt another phase of a design process. Especially when it involve the concept development of Product Architecture, which determine by product change, product variety, component standardization, product performance and product development management [37]. Through our analysis, the design structure(syntactic) formed during the abstraction level (Table 3a, 3b, and 3c) has summarized, it is important to structure explicitly the ablution to occupied the need to perform the principles (face, both hand, forehead and both feet). However, the designer in such a way has practical means on the forming surface of the form (design structure) in representing the influence of their intuition mind strategy. The only visible element used as an explicit design is the arabesque design. Three level of abstraction implies this element to represent the product was initiated by Islamic influence.

We hypotheses that the activities of interaction with influence panel make the designer matured with a stronger embodied understanding of the form structure. This IVDP experiment also shows that designer interacts the mental image with eye contact and experience in designers' apparently intuitive formgiving process. The designer has played with particular 'configuration system' in an intuitive stage to understand and visualize form related to the image in the mind. Function Mean Analysis here uses to identify on, how well the designer knows when is the right time to change from one domain to the other. The transformation of abstraction level(through designer's think and working drawing) clearly through conceptual and embodiment phases represents a product-based system, and allowing us to decompose the analysis system due to the similarity in term of design outcomes. 


\section{Conclusions}

In conclusion, we strongly disagree if creative work cannot be considered as research and only taking apart of observable phenomena. Cross, in his taxonomy, agree that three main categories should be a part of design research which based on people, process, and products. In our first finding, the design practices or topics mostly have a strong connection to design research. Three out of five research topic mentioned used by the designer as key metaphor design categories. IVDP as artificial situation strategy with well structure design brief has stated clear direction of design task and how designer's think while creating a creative work. The designer where in this study we call as 'subject' has proved that design development constructed during 'abstract' 'semi-concrete' and 'concrete' level, often close to 'goal-oriented problem-solving activity' as defined by Archer. It shows that even creative designer can offer a systematic investigation, especially through IVDP. Novel facts, using a scientific method such IVDP may provide a new method of design research especially in the field of art and design.

\section{Acknowledgement}

We would like to acknowledge the generous participation of the interaction designers in the research. This study was conducted in Formgiving Design Research Lab, UiTM. Fully appreciation to Malaysia Ministry of Higher Education for the financial support under RAGS and UiTM for the REI grant.

\section{References}

1. S. Z. Abidin, D. Christoforidou, and A. Liem. Thinking And Re-Thinking Verbal Protocol Analysis In Design Research. Stanford: International Conference On Engineering Design, pp.112(Aug. 2009).

2. R. Anwar, S. Z. Abidin, and O. H. Hassan. A Framework of Empirical Study Through Design Practice for Industrial Ceramic Sanitary Ware Design. In ICADER 2014 by O. H. Hassan, S. Z. Abidin, R. Legino, R. Anwar and M. F. Kamaruzaman (eds). Singapore: Springer (2015).

3. R. C. Valkenburg, Shared understanding as a condition for team design." Automation in Construction, vol. 7 (2), pp. 111-121 (1998).

4. B. Sener, and P. Wormald. User evaluation of HCI concepts for defining product form. Design Studies, vol. 29 (1), pp. 12-29(2007).

5. G. Pahl, and W Beitz. Engineering design. London: The Design Council and Springer Verlag(1984).

6. S. Pugh, D. Clausing, and R. Andrade. Creating innovative products using total design. New York: Addison-Wesley Publishing (1996).

7. R. Anwar, S. Z. Abidin, and O. H. Hassan. Function Means Analysis For Ablution Concept Solution. The Turkish Online Journal of Educational Technology, pp. 224-231(2015).

8. N. Cross. Engineering Design Methods: Strategies for Product Design. Chichester: John Wiley \& Sons, Ltd (2000).

9. L. B. Archer. Systematic Method for Designers. In Developments in Design Methodology, by Nigel Cross (eds). Chichester: John Wiley \& Sons(1984).

10. S. Pugh. Total design: integrated methods for successful product engineering. Boston: AddisonWesley(1991).

11. L. B. Archer. A View of the Nature of the Design Research. In Design: Science: Method, by R. Jacques and J. A. Powell, Guilford, Surrey: IPC Business Press Ltd., pp. 30-47(1981).

12. C. Frayling. Research in art and design. vol. 1 (1), London: Royal College of Art Research Papers(1993).

13. C. L. Owen. Design research, building the knowledge base. Design Processes Newsletter 5 no. 6, pp. 1-6(1994).

14. N. Bayazit. Investigating Design: A Review of Forty Years of Design Research.Design Issues 20 (1), pp. 16-29(2004). 
15. S. Z. Abidin, J. Sigurjonsson, A. Liem, and M. Keitsch. On the Role of Formgiving in Design. International Conference On Engineering \& Product Design Education. Barcelona, pp. 365370(Sept. 2008).

16. N. Cila, P. Hekkert, and V. Visch. Source Selection in Product Metaphor Generation: The Effects of Salience and Relatedness. International Journal of Design, vol. 1 (2), pp. 15-28(2014).

17. R. L. Marsh, T. B. Ward, and J. D. Land. The inadvertent use of prior knowledge in a generative cognitive task. Memory \& Cognition. vol 27 (1), pp. 94-105(1999).

18. S. Z. Abidin, H. V. Bjelland, and T. A. Øritsland. The embodied mind in relation to thinking about form development. Proceedings of NordDesign 2008 Conference. Tallin: pp. 265-274, Aug. 2008.

19. G. Lakoff and M. Johnson. Philosophy in the Flesh The Embodied Mind and Its Challenge to Western Thought. New York: Basic Book, 1999.

20. K. Dorst. The Core of 'Design Thinking' and Its Application. Design Studies. vol. 32 (6), pp. 521-532(2011).

21. H. P. Casakin. Factors of metaphors in design problem-solving: Implications for design creativity. International Journal of Design. vol. 1 (2), pp. 21-33(2007).

22. G. Pahl, W. Beitz, J. Feldhusen, and K. Grote. Engineering Design: A Systematic Approach. 3rd. Edited by K. Wallace and L. T. M. Blessing. London: Springer-Verlag(2007).

23. R. Anwar, S. Z. Abidin, and O. H. Hassan. A Pattern in Formgiving Design: Giving Priority to a Principle Solution in Industrial Design Situation. In Industrial Engineering, Management Science and Applications 2015, by M. Gen, K. J. Kim, X. Huang and Y. Hiroshi, Berlin Heidelberg: Springer, pp. 331-340(2015).

24. N. Cross. Designerly Ways of Knowing. London: Springer-Verlag(2006).

25. V. Hubka, M. M.Andreasen, and W. E. Eder. Practical Studies in Systematic Design. Edited by Peter Hills. London: Butterworths(1988).

26. E. Tjalve. Systematic Design of Industrial Product. Lyngby: Institute for Product Development(2003).

27. S. Z. Abidin. Practice-based design thinking for form development and detailing. PhD Thesis. Trondheim: Norwegian University of Science and Technology, 2012.

28. L. T. M. Blessing. A process-based approach to computer-supported engineering design. $\mathrm{PhD}$ thesis. Twente: University of Twente, 1994.

29. R. Anwar, S. Z. Abidin, and O. H. Hassan. Understanding Methodological Solution In Design Situation Of Novice Designer. 18th International Conference on Interactive Collaborative Learning, Florence, IEEE. pp. 593-597 (Sept. 2015).

30. R. Anwar, S. Z. Abidin, and O. H. Hassan. The Turkish Online Journal of Educational Technology, pp. 13-21(2015).

31. N. H. Johari, R. Anwar, and O. H. Hassan. "Design framework of ceramic ablution Tub." IEEE Symposium on Business, Engineering and Industrial Applications, Bandung, IEEE, pp. 608610(2012).

32. S. Z. Abidin, A. Warell, and A. Liem. Understanding Styling Activity Of Automotive Designers: A Study Of Manual Interpolative Morphing Through Freehand Sketching. Proceedings of the 18th International Conference on Engineering Design (ICED11). Copenhagen: Design Society, pp. 357-366(Aug. 2011).

33. W. Muller. Order and Meaning in Design. Utrecth: Lemma Publisher(2001).

34. A. Warell. Design Syntactics: A Functional Approach to Visual Product Form. Goteborg: Chalmers University of Technology(2001).

35. R. Mono. Design for Product Understanding: The Aesthetics of Design from a Semiotic Approach. Stockholm: Liber AB(1997).

36. A. Liem. Managing The Industrial Design Process: A Guide for Studio Practice. Singapore: Prentice Hall(2004).

37. K.T. Ulrich and S.D. Eppinger. Product Design and Development, 5th, New Delhi: TATA McGraw-Hill Edition(2005). 